\title{
Analysis of Buckling Characteristics and Parameter Influence of Composite Thin-walled Lenticular Boom Structures
}

\author{
Zhichao Yao ${ }^{1}$, Dongxu Li ${ }^{1, *}$, Shiyao Zhu ${ }^{1}$, Lilin Zhou ${ }^{1}$ \\ ${ }^{1}$ College of Aerospace Science and Engineering, National University of Defense Technology, 410073 Changsha, China
}

\begin{abstract}
The stretchable composite thin-walled lenticular boom can be used in the unfolding process of a large spacecraft structure, and its buckling characteristic is one of the focuses of structural design. In this paper, firstly, the critical buckling load formula is derived based on Euler's formula and laminated theory for the axial compression buckling problem of the lenticular boom, and verified by the finite element method. Secondly, the influence law of the lenticular boom section and layer parameters on the critical buckling load is quantitatively analyzed. The results show that the lenticular boom generally undergoes first-order buckling in the outer direction of the symmetrical bonding surface. The critical buckling load is most significantly affected by the radius of the convex arc, followed by the center ordinate of the convex arc, the thickness of the layer, and the angle of the layer. And these parameters are positively related to the critical buckling load. The radius of the concave arc and the length of the straight section have little effect on the critical buckling load. The research methods and conclusions of this paper can provide reference for the engineering design of the lenticular boom structure.
\end{abstract}

\section{Introduction}

The thin-walled lenticular boom structure was first designed and developed by the German Aerospace Center ${ }^{[1]}$. It is a space-expandable structure made of carbon fiber reinforced composite materials, named after its similar cross-sectional shape. The lenticular boom has the performance characteristics of light weight, high rigidity, high storage rate. So it can be used in the stretching process of large spacecraft structures ${ }^{[2]}$. The lenticular boom plays a supporting role during the deployment of a large spacecraft ${ }^{[3]}$. Due to the slender configuration of the lenticular boom, it is prone to buckling damage under the action of axial pressure, which affects its bearing capacity, so domestic and foreign scholars have conducted research on it.

Abroad, Sickinger et al. ${ }^{[4]}$ studied the structural failure of the lenticular boom under axial compression. Herbeck et al. ${ }^{[5]}$ prepared a 14-meter-long lenticular boom model and conducted ground tests to obtain the linear and nonlinear flexural rigidity and buckling load of the lenticular boom. Hakkak et al. ${ }^{[6]}$ theoretically deduced the elastic strain energy of the lenticular boom after being compressed, and conducted a stress analysis on the lenticular boom under bending and torsion load in the unfolded state. Christian [7] applied numerical methods to study the buckling and post-buckling behaviors of thin-wall composite laminated beams. Irwin et al. ${ }^{[8]}$ obtained the flexural rigidity and bearing capacity of the lenticular boom through a four-point bending test. In China, $\mathrm{Hu}$ et al. ${ }^{9]}$ studied the modal behavior of the lenticular boom under free vibration and cantilever vibration through experiments and numerical methods, and carried out the linear and nonlinear buckling analysis of the lenticular boom under axial compression. Li et al. ${ }^{[10]}$ conducted axial compression load tests and numerical simulations on a four-layer lenticular boom structure, obtained critical buckling load test values and numerical solutions under axial compression conditions, and compared different composite material layup methods. The influence of lenticular boom's cross-section and layering parameters on buckling characteristics is not systematically analyzed. Chu et al. ${ }^{[11]}$ analyzed the bending stress and torsion stress of the lenticular boom through theoretical derivation, and used the sequential quadratic programming method to optimize the parameters of the lenticular boom with stress and natural frequency as the optimization objective. Bai et al. [12] studied the influence of temperature on the axial compression buckling performance of lenticular boom, and compared the experimental results with the numerical simulation results. Guo et al. ${ }^{[13]}$ analyzed the changes in energy, external load and stiffness of the lenticular boom during the flattening process.

It can be seen from the above research that the finite element analysis and experimental research on the axial compression buckling of the lenticular boom are abundant, but the theoretical analysis of the axial compression buckling of the composite lenticular boom is lacking. The research on the influence law of the cross-sectional geometric parameters of the lenticular boom and the parameters of the composite layer is not systematic enough. In response to this problem, this

\footnotetext{
* Corresponding author: dongxuli@nudt.edu.cn
} 
paper first derives the critical buckling load formula of composite lenticular boom based on Euler's formula and laminated theory, verifies its correctness by finite element method, and discusses the applicable scope of the formula. Then quantitatively analyze the influence of cross-section geometric parameters and composite layer parameters on critical buckling load. The analysis methods and results in this paper have certain reference significance for the structural engineering design of composite thin-walled lenticular boom.

\section{Theoretical analysis of the buckling problem of lenticular boom}

\subsection{General Euler instability problem}

The boundary condition is that a slender rod with one end fixedly supported and one end free is prone to buckling instability and loss of working ability under the action of axial pressure. Aiming at the buckling problem of compression rods, Eluer put forward the following hypotheses:

(1) The elongated rod is made of isotropic material;

(2) The rod axis is a straight line, and the pressure line of action coincides with the rod axis;

(3) When buckling occurs, the stress does not exceed the proportional limit of the material;

(4) When buckling occurs, the deformation of the rod is small deformation.

Under the above assumptions, Eluer derived the critical buckling load formula for a slender compression rod with one end fixed and one end free:

$$
F_{c r}=\frac{\pi^{2} E I_{\min }}{(2 l)^{2}}
$$

Where: $E$ is the elastic modulus, $\mathrm{I}_{\text {min }}$ is the minimum moment of inertia of the slender rod cross section, and $l$ is the rod length. As shown in Figure 1, when the applied load reaches the critical buckling load, the slender rod buckles ${ }^{[14]}$.

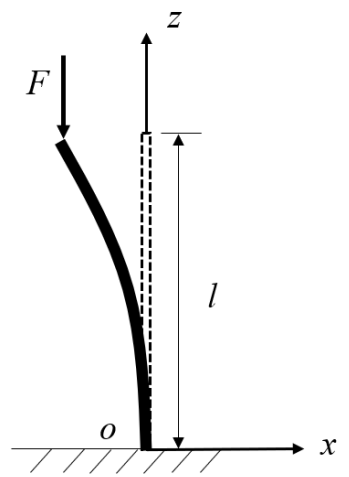

Fig. 1. Euler instability diagram

\subsection{The theoretical modelling of lenticular boom}

The lenticular boom plays a supporting role during work, and the supported object exerts axial pressure on the lenticular boom. At this time, the lenticular boom is prone to buckling and instability. Assuming that the axis of the lenticular boom is a straight line, and the pressure line of action coincides with the axis; when the lenticular boom buckles, the stress does not exceed the proportional limit of the material, and the deformation of the lenticular boom is a small deformation.

As shown in Figure 2(a), the lenticular boom under axial pressure is simplified as a straight rod under pressure, and the boundary condition is that one end is fixed and the other end is free. The lenticular boom is a thin-walled tube formed by glueing two " $\Omega$ "-shaped half lenticular boom at the boundary. The cross-sectional schematic diagram is shown in Figure 2(b). The origin $o$ of the coordinate system is taken at the geometric center of the connecting surface of the lenticular boom and the spacecraft body, and the $o x$ axis passes through the glued surface of the lenticular boom section. The $o z$ axis coincides with the neutral axis of the lenticular boom and points to the extension direction of the lenticular boom. The $o y$ axis is determined by the right hand rule. It can be known from the mechanics of materials that since the cross section of the lenticular boom is symmetric about the $o x$ axis and the $o y$ axis, the product of inertia of the cross-section $I_{x y}=0$. Because the origin $o$ is the centroid of the lenticular boom section, the $o x$ axis and $o y$ axis are the centroid main inertia axes ${ }^{[15]}$. Therefore, the lenticular boom will buckle around the $o x$ axis or $o y$ axis.

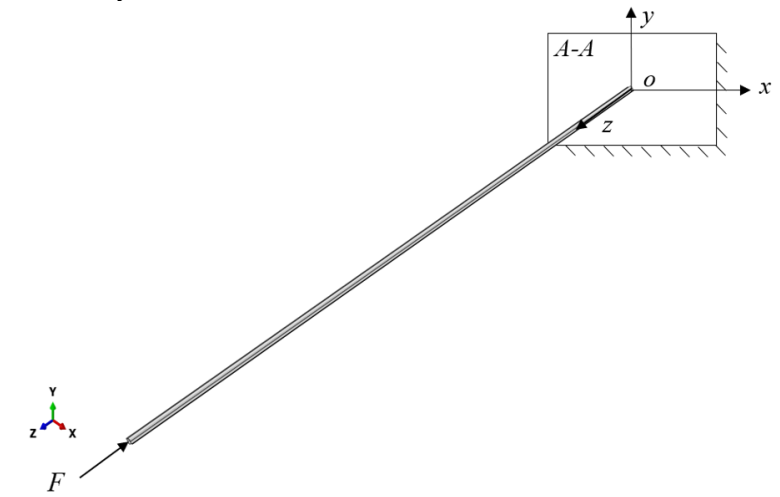

(a) Simplified model and coordinate system of lenticular boom

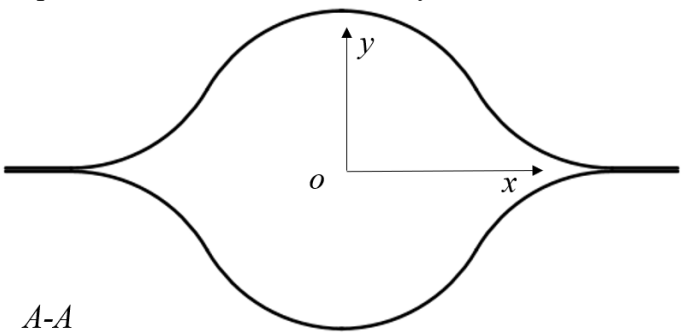

(b) Schematic diagram of $A-A$ section

Fig. 2. Simplified model and cross-sectional schematic diagram of lenticular boom

It can be seen that the buckling problem of the lenticular boom satisfies the basic assumptions (2), (3) and (4) of the general Euler instability problem. Therefore, in view of the geometric configuration and the characteristics of the composite material layer of the lenticular boom, this paper derives the critical buckling load formula of the lenticular boom on the basis of Euler's formula, as follows: 


$$
F_{c r}^{*}=\frac{\pi^{2}}{(2 l)^{2}} \cdot \sum_{i=1}^{n} E_{z, i} I_{i}
$$

Among them, based on the geometric configuration and the characteristics of the composite material layer of the lenticular boom, the cross section of the lenticular boom can be divided into $n$ sections, and the axial equivalent elastic modulus $E_{z, i}$ and the moment of inertia $I_{i}$ of the i-th section can be solved and superimposed. Finally, the overall critical buckling load of the lenticular boom is obtained.

In this article, the cross section of the lenticular boom is symmetrical about the $o x$ axis and the $o y$ axis, and the origin $o$ is the geometric center of the cross section, so a quarter of the cross section of the lenticular boom can be used for variable definition and geometric relationship description. As shown in Figure 3, the quarter crosssection of the lenticular boom can be divided into 3 sections $(n=12)$, namely the convex arc, the concave arc and the straight section, which are represented by $A B$, $B C$ and $C D$ respectively. And the leftmost tangent of the convex arc is perpendicular to the oy axis, the convex arc is tangent to the concave arc, and the concave arc is tangent to the straight section. $r_{1}$ and $r_{2}$ are the radius of convex arc and concave arc respectively. $\alpha_{1}$ and $\alpha_{2}$ are the corresponding arc angles of convex and concave arcs, respectively. $\left(x_{1}, y_{1}\right)$ and $\left(x_{2}, y_{2}\right)$ are the center coordinates of the convex arc and the concave arc respectively. $h$ is the total thickness of the arc, and the parameter subscripts " 1 ", " 2 " and " 3 " respectively represent the convex arc, the concave arc and the straight section. $w$ is the length of the straight section.

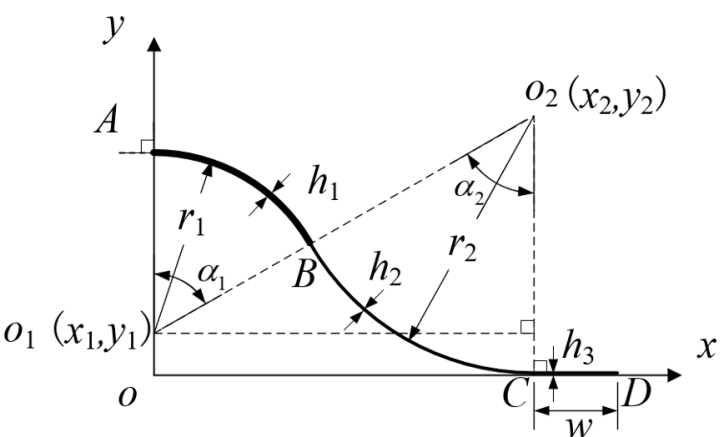

Fig. 3. Schematic diagram of the quarter cross-sectional shape of the lenticular boom

According to the geometric relationship, in addition to the thickness, the independent parameters of the crosssectional shape include $r_{1}, r_{2}, w$ and $y_{1}$. The independent variables $\alpha, x_{1}, x_{2}$ and $y_{2}$ can be expressed as:

$$
\left\{\begin{array}{l}
\alpha=\alpha_{1}=\alpha_{2}=\arccos \frac{r_{2}-y_{1}}{r_{1}+r_{2}} \\
x_{1}=0 \\
x_{2}=\left(r_{1}+r_{2}\right) \sin \alpha \\
y_{2}=r_{2}
\end{array}\right.
$$

According to the characteristics of the cross-sectional geometric shape of the lenticular boom, select the infinite element $\mathrm{d} s_{i}$ on the arc/straight line segment. $\mathrm{d} \theta_{i}$ is the angle corresponding to the infinite element $\mathrm{d} s_{i}$, and there is $\mathrm{d} s_{i}=r_{i} \mathrm{~d} \theta_{i}$. In the arc length direction integral calculation, the moments of inertia of each arc/straight line segment around the $o x$ axis are:

$$
\begin{aligned}
& I_{x, 1}=\int_{A B} y^{2} h_{1} \mathrm{~d} s_{1} \\
& =\int_{0}^{\alpha}\left(y_{1}+r_{1} \cos \theta_{1}\right)^{2} r_{1} h_{1} \mathrm{~d} \theta_{1} \\
& =r_{1} h_{1}\left(r_{1}^{2}\left(\frac{1}{4} \sin 2 \alpha+\frac{1}{2} \alpha\right)+y_{1}^{2} \alpha+2 y_{1} r_{1} \sin \alpha\right) \\
& I_{x, 2}=\int_{B C} y^{2} h_{2} \mathrm{~d} s_{2} \\
& =\int_{0}^{\alpha}\left(r_{2}-r_{2} \cos \theta_{2}\right)^{2} h_{2} r_{2} \mathrm{~d} \theta_{2} \\
& =r_{2}^{3} h_{2}\left(\frac{1}{4} \sin 2 \alpha-2 \sin \alpha+\frac{3}{2} \alpha\right) \\
& I_{x, 3}=\frac{1}{2} \cdot \frac{w\left(2 h_{3}\right)^{3}}{12}=\frac{w h_{3}^{3}}{3}
\end{aligned}
$$

In the same way, the moments of inertia of each arc/straight segment around the $o y$ axis are derived as:

$$
\begin{aligned}
& I_{y, 1}=\int_{A B} x^{2} h_{1} \mathrm{~d} s_{1} \\
&=\int_{0}^{\alpha}\left(r_{1} \sin \theta_{1}\right)^{2} r_{1} h_{1} \mathrm{~d} \theta_{1} \\
&=r_{1}^{3} h_{1}\left(\frac{1}{2} \alpha-\frac{1}{4} \sin 2 \alpha\right) \\
& I_{y, 2}=\int_{B C} x^{2} h_{2} \mathrm{~d} s_{2} \\
&=\int_{0}^{\alpha}\left(\sqrt{\left(r_{1}+r_{2}\right)^{2}-\left(r_{2}-y_{1}\right)^{2}}-r_{2} \sin \theta_{2}\right)^{2} r_{2} h_{2} \mathrm{~d} \theta_{2} \\
&=r_{2} h_{2}\left(\left(r_{1}^{2}+2 r_{1} r_{2}-y_{1}^{2}+2 r_{2} y_{1}+\frac{1}{2} r_{2}^{2}\right) \alpha-\frac{1}{4} r_{2}^{2} \sin 2 \alpha\right) \\
& I_{y, 3}=\frac{1}{2}\left(\frac{2 h_{3} w^{3}}{12}+2 h_{3}^{2}+2 r_{1} r_{2}-y_{1}^{2}+2 r_{2} y_{1}\left(\sqrt{r_{1}^{2}+2 r_{1} r_{2}-y_{1}^{2}+2 r_{2} y_{1}}+\frac{w}{2}\right)^{2}\right) \\
&= h_{3}\left(\frac{w^{3}}{12}+w\left(\sqrt{r_{1}^{2}+2 r_{1} r_{2}-y_{1}^{2}+2 r_{2} y_{1}}+\frac{w}{2}\right)^{2}\right)
\end{aligned}
$$

According to the laminated theory ${ }^{[15]}$, the equivalent elastic modulus of the composite laminate along the axial direction of the lenticular boom is:

$$
E_{z, i}=\frac{1}{h_{i}} \frac{A_{11, i} A_{22, i}-A_{12, i}^{2}}{A_{22, i}} \quad i=1,2,3
$$

In formula (10), $A_{11, i}, A_{22, i}$ and $A_{12, i}$ are the stiffness coefficients between the pressure and the mid-plane compressive strain, and the expression is as follows: 


$$
\left\{\begin{array}{l}
A_{11, i}=t \sum_{k=1}^{N_{i}}\left(Q_{11} \cos ^{4} \beta_{k}+2\left(Q_{12}+2 Q_{66}\right) \sin ^{2} \beta_{k} \cos ^{2} \beta_{k}+Q_{22} \sin ^{4} \beta_{k}\right) \\
A_{22, i}=t \sum_{k=1}^{N_{i}}\left(Q_{11} \sin ^{4} \beta_{k}+2\left(Q_{12}+2 Q_{66}\right) \sin ^{2} \beta_{k} \cos ^{2} \beta_{k}+Q_{22} \cos ^{4} \beta_{k}\right) \\
A_{12, i}=t \sum_{k=1}^{N_{i}}\left(\left(Q_{11}+Q_{22}-4 Q_{66}\right) \sin ^{2} \beta_{k} \cos ^{2} \beta_{k}+Q_{12}\left(\sin ^{4} \beta_{k}+\cos ^{4} \beta_{k}\right)\right)
\end{array}\right.
$$

In formula (11), $t$ is the thickness of the single-layer composite material; $\beta_{k}$ is the ply angle of the $k$-th layer in the laminate; $N_{i}$ is the total number of plies; and $Q_{11}, Q_{22}, Q_{12}$ and $Q_{66}$ are the plane stress modulus under the positive axis. The expression is as follows:

$$
\left\{\begin{array}{l}
Q_{11}=\frac{E_{1}}{1-\mu_{12} \mu_{21}} \\
Q_{22}=\frac{E_{2}}{1-\mu_{12} \mu_{21}} \\
Q_{12}=\frac{\mu_{12} E_{2}}{1-\mu_{12} \mu_{21}}=\frac{\mu_{21} E_{1}}{1-\mu_{12} \mu_{21}} \\
Q_{66}=G_{12}
\end{array}\right.
$$

Substituting formula (4) to (10) into formula (2), the critical buckling loads of the lenticular boom around the $o x$ axis and the $o y$ axis are:

$$
\begin{aligned}
F_{c r, x}^{*} & =\frac{\pi^{2}}{(2 l)^{2}} \cdot 4\left(E_{z, 1} I_{x, 1}+E_{z, 2} I_{x, 2}+E_{z, 3} I_{x, 3}\right) \\
& =\frac{\pi^{2}}{l^{2}}\left(E_{z, 1} I_{x, 1}+E_{z, 2} I_{x, 2}+E_{z, 3} I_{x, 3}\right) \\
F_{c r, y}^{*} & =\frac{\pi^{2}}{(2 l)^{2}} \cdot 4\left(E_{z, 1} I_{y, 1}+E_{z, 2} I_{y, 2}+E_{z, 3} I_{y, 3}\right) \\
& =\frac{\pi^{2}}{l^{2}}\left(E_{z, 1} I_{y, 1}+E_{z, 2} I_{\mathrm{y}, 2}+E_{z, 3} I_{y, 3}\right)
\end{aligned}
$$

In summary, the theoretical critical buckling load of the lenticular boom is the minimum of the critical buckling load for about the $o x$ axis and the critical buckling load for about the oy axis, that is:

$$
F_{c r}^{*}=\min \left(F_{c r, x}^{*}, F_{c r, y}^{*}\right)
$$

\section{Finite element simulation analysis of buckling of lenticular boom}

\subsection{Finite element modeling of lenticular boom}

The cross-sectional parameters of the lenticular boom in this buckling analysis are shown in Table 1. The upper and lower parts of the lenticular boom are bonded by the resin bonding layer. The single-layer composite material parameters are shown in Table 2, and the rod length is $l=6.5 \mathrm{~m}$. A schematic diagram of the lenticular boom layering is shown in Figure 4, and the $0^{\circ}$ direction of the composite material layer is the axial direction of the lenticular boom. The number of layers of the convex arc composite material is 7 layers, the thickness of each layer is $0.04 \mathrm{~mm}$, and the layer angle is $[45 \%$ $45^{\circ} / 0^{\circ} / 0^{\circ} / 0^{\circ} / 45^{\circ} / 45^{\circ}$ ]. Compared with the convex arc, the concave arc and the straight section lack the third and fifth layers. The bonding layer is used as the eighth layer, and the material parameters are shown in Table 3.

Table 1. Lenticular boom section parameters.

\begin{tabular}{|c|c|}
\hline Parameter & Value \\
\hline$r_{1}(\mathrm{~mm})$ & 20.00 \\
\hline$r_{2}(\mathrm{~mm})$ & 20.00 \\
\hline$w(\mathrm{~mm})$ & 5.00 \\
\hline$\alpha\left({ }^{\circ}\right)$ & 60.00 \\
\hline$y_{1}(\mathrm{~mm})$ & 0.00 \\
\hline$h_{1}(\mathrm{~mm})$ & 0.28 \\
\hline$h_{2}(\mathrm{~mm})$ & 0.20 \\
\hline$h_{3}(\mathrm{~mm})$ & 0.24 \\
\hline
\end{tabular}

Table 2. Single layer composite material parameters.

\begin{tabular}{|c|c|}
\hline Parameter & Value \\
\hline Density $\left(\mathrm{kg} / \mathrm{m}^{3}\right)$ & 1505.00 \\
\hline Longitudinal tensile modulus $(\mathrm{GPa})$ & 82.30 \\
\hline Transverse tensile modulus $(\mathrm{GPa})$ & 6.77 \\
\hline Poisson's ratio & 0.33 \\
\hline Shear modulus $(\mathrm{GPa})$ & 3.50 \\
\hline
\end{tabular}

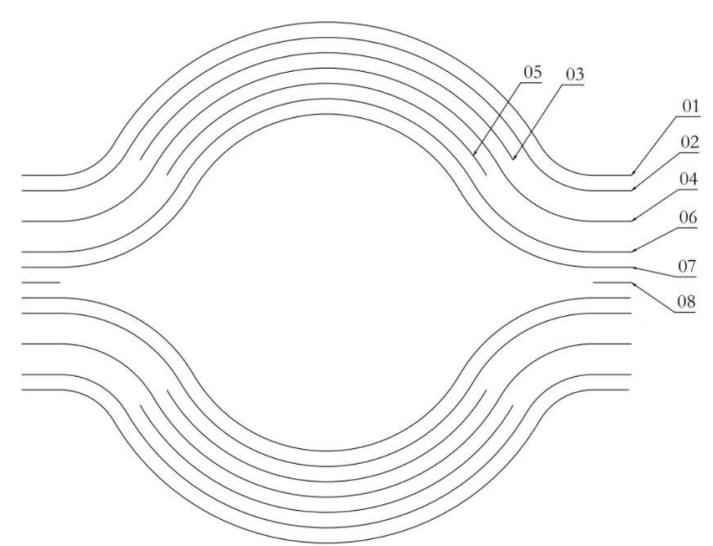

Fig. 4. Schematic diagram of the lenticular boom layer

Table 3. Material parameters of resin bonding layer.

\begin{tabular}{|c|c|}
\hline Parameter & Value \\
\hline Density $\left(\mathrm{kg} / \mathrm{m}^{3}\right)$ & 1250.00 \\
\hline Tensile modulus $(\mathrm{GPa})$ & 3.02 \\
\hline Poisson's ratio & 0.33 \\
\hline
\end{tabular}

The ABAQUS software is used for finite element modeling analysis, and the shell element is selected for modeling according to the shape characteristics of the lenticular boom. Shell element types commonly used in ABAQUS include S8R, S4R, and S4 elements. Among them, the S4R thin shell element is based on Mindlin theory, with hourglass control and Kirchhoff condition (transverse shear strain is zero). In the process of calculation and analysis, it has the characteristics of high 
calculation efficiency, strong robustness, and good convergence. So it is the first choice for thin-shell modeling ${ }^{[16]}$. In this paper, the lenticular boom belongs to a thin shell structure, so S4R elements are selected for meshing, and the arcs are divided into sufficiently fine meshes. The finite element model of the lenticular boom is shown in Figure 5. A reference point is created at the center of the free end of the lenticular boom, and the reference point is constrained with the free end section to apply the load. For the restraint method of the reference point and the free end section, MPC constraints and coupling constraints are both possible. However, after simulation analysis, it is found that after the introduction of MPC constraints, the degree of freedom of the rotation direction of the free end will be additionally restricted, which is not suitable for relaxing the boundary conditions of the rotation limit. However, when the motion coupling constraint is introduced, there will be no additional constraint degrees of freedom. Therefore, the motion coupling constraint method is selected to constrain the reference point and the free end section.

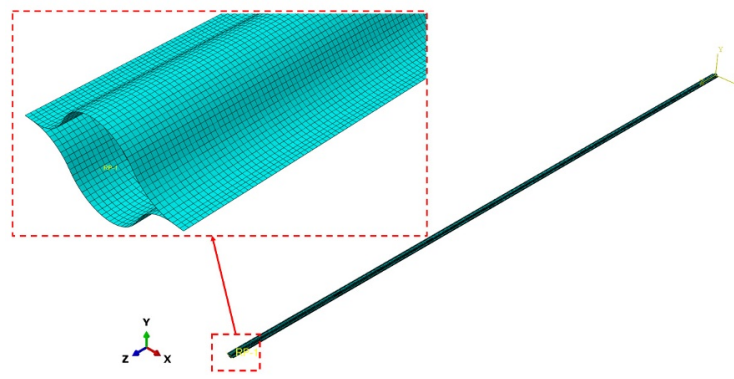

Fig. 5. Finite element model of lenticular boom

The eigenvalue buckling analysis of the lenticular boom structure is carried out. Create a buckling analysis step in ABAQUS and apply $1 \mathrm{~N}$ pressure on the free end of the lenticular boom. Figure 6 shows the first 2 buckling modes of the lenticular boom through calculation. It can be seen that the first-order buckling mode is the case of buckling around the ox axis, and the second-order buckling mode is the case of buckling around the oy axis. Due to the fact that the fiber shape and size of the actual composite material are not completely regular and there are defects in the material, it is necessary to use the Riks method to perform nonlinear buckling analysis of the lenticular boom structure ${ }^{[17]}$. Introducing the initial geometric defects in the analysis, it is necessary to determine the defect factor and the introduced buckling mode to provide a buckling path when the structure is unstable.

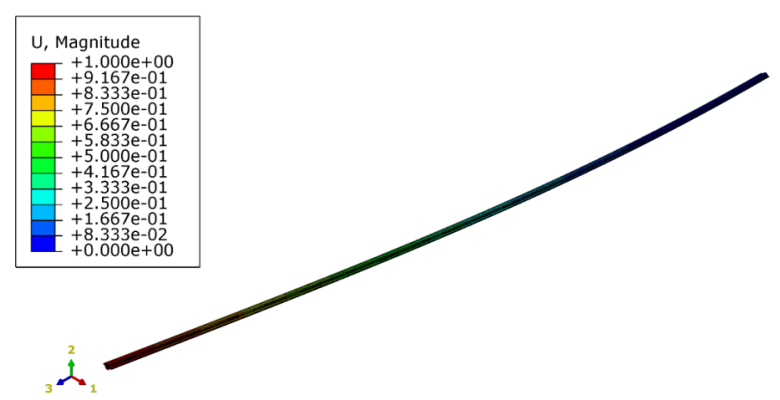

(a) The first-order buckling mode

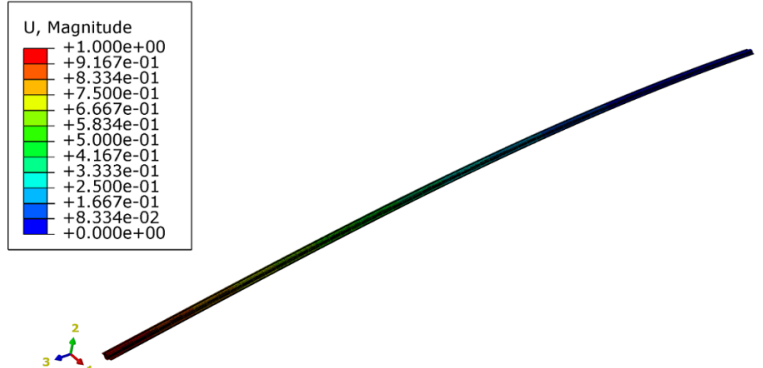

(b) The second-order buckling mode

Fig. 6. The first 2 buckling modes of the lenticular boom under axial compression

\subsection{Comparison of theoretical deduction and finite element analysis results}

The comparison of results obtained by the theoretical method, eigenvalue buckling method and nonlinear method is shown in Table 4, where the defect factor in the nonlinear buckling analysis is $0.1 \mathrm{~mm}$. The analysis results show that the three critical buckling loads agree well, which verifies the accuracy of the critical buckling load formula for the composite lenticular boom. Since the section deformation and initial geometric defects during buckling are ignored in the theoretical analysis, the theoretical critical buckling load will be a little larger. In the eigenvalue buckling analysis, the section deformation is considered, but the initial geometric defects are ignored, so the eigenvalue critical buckling load will be slightly smaller than the theoretical buckling load. In nonlinear buckling analysis, section deformation and initial geometric defects are considered, so the nonlinear critical buckling load will be more accurate and smaller than the first two critical buckling loads.

Table 4. Comparison of results of three analysis methods.

\begin{tabular}{|c|c|c|c|}
\hline $\begin{array}{c}\text { Critical } \\
\text { buckling } \\
\text { load }\end{array}$ & $\begin{array}{c}\text { Theoretica } \\
\text { I analysis }\end{array}$ & $\begin{array}{c}\text { Eigenvalue } \\
\text { buckling } \\
\text { analysis }\end{array}$ & $\begin{array}{c}\text { Nonlinear } \\
\text { buckling } \\
\text { analysis }\end{array}$ \\
\hline $\begin{array}{c}\text { Buckling } \\
\text { around the } \\
\text { ox axis(N) }\end{array}$ & $\mathbf{1} 7.07$ & 17.03 & 16.84 \\
\hline Difference & $/$ & $-0.23 \%$ & $-1.35 \%$ \\
\hline $\begin{array}{c}\text { Buckling } \\
\text { around the } \\
\text { oy axis(N) }\end{array}$ & 32.58 & 32.57 & 32.32 \\
\hline Difference & $/$ & $-0.03 \%$ & $-0.68 \%$ \\
\hline
\end{tabular}

Since the section deformation during buckling is neglected in the derivation process of this paper, it is necessary to discuss the applicable range of the critical buckling load formula of lenticular boom. Define the ratio of the length of the lenticular boom to the radius of gyration of the section as the slenderness ratio $\lambda$. The relationship between $\lambda$ and the buckling load obtained through theoretical analysis and eigenvalue analysis is shown in Figure 7 . When $\lambda$ is small, the theoretical analysis result deviates greatly from the eigenvalue 
analysis result. For example, when $\lambda$ is 40 , the deviations of the analysis results of $F_{c r, x}^{*}$ and $F_{c r, y}^{*}$ are $13.13 \%$ and $-54.10 \%$, respectively. As $\lambda$ gradually increases, the deviation of the analysis results gradually decreases. For example, when $\lambda$ is greater than 64 , the deviation between the theoretical analysis result and the eigenvalue analysis result is less than $1 \%$. Therefore, when the $\lambda$ of the lenticular boom is greater than 64 , the critical buckling load formula is applicable.

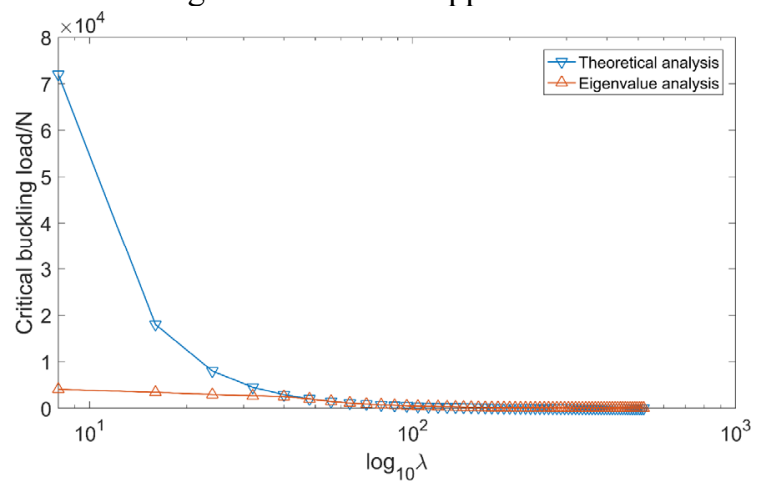

(a) Buckling around the $o x$ axis

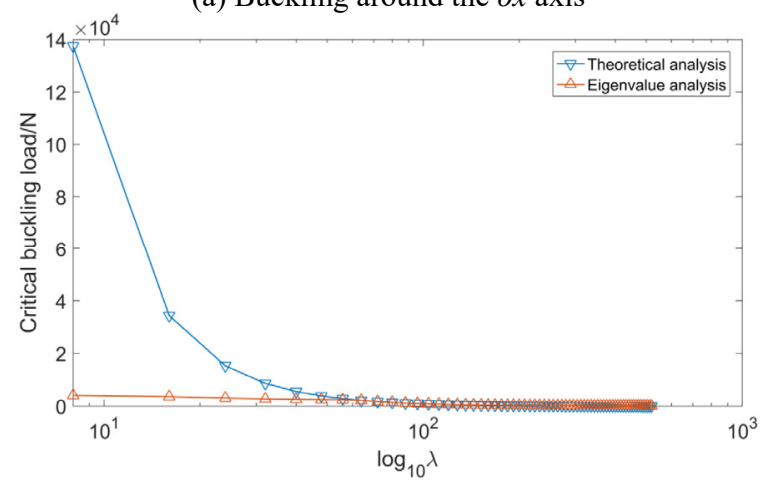

(b) Buckling around the oy axis

Fig. 7. The influence of $\lambda$ on critical buckling load

\section{Parameter analysis of critical buckling load formula for lenticular boom}

Based on the critical buckling load formula of the lenticular boom, the influence of the cross-sectional geometric parameters and composite layer parameters on the critical buckling load of the lenticular boom are quantitatively analyzed.

\subsection{The influence of section geometrical parameters on critical buckling load}

\subsubsection{The influence of the radius of the convex arc on critical buckling load}

Based on the original parameters of the model, expanding the value of $r_{1}$ from $20 \mathrm{~mm}$ to $10 \mathrm{~mm} \sim 30 \mathrm{~mm}$. Figure 8 shows the change of critical buckling load when $r_{1}$ changes. $r_{1}$ has a positive correlation with the critical buckling load. When $r_{1}$ increases and decreases by $50 \%$, $F_{c r, x}^{*}$ increases by $241.83 \%$ and decreases by $87.94 \%$, and $F_{c r, y}^{*}$ increases by $150.31 \%$ and decreases by $71.96 \%$, respectively. As $r_{1}$ increases, the slope of the curve between $r_{1}$ and critical buckling load becomes larger. Therefore, increasing $r_{1}$ can effectively increase the critical buckling load and enhance the buckling resistance of the lenticular boom.

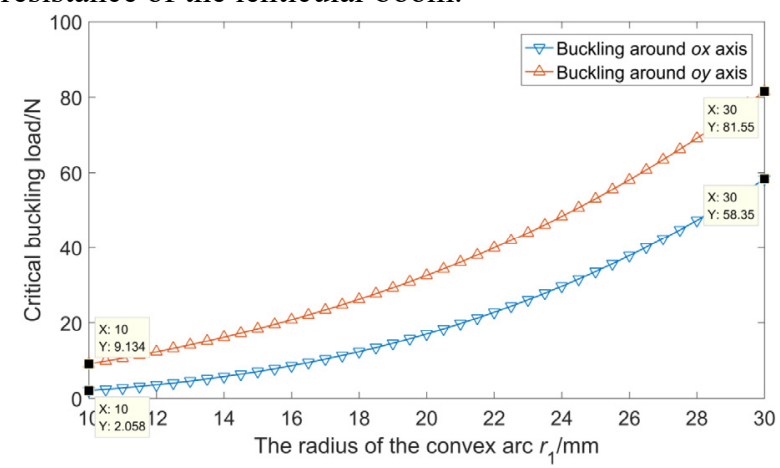

Fig. 8. The influence of $r_{1}$ on critical buckling load

\subsubsection{The influence of the radius of the concave arc on critical buckling load}

Based on the original parameters of the model, expanding the value of $r_{2}$ from $20 \mathrm{~mm}$ to $10 \mathrm{~mm} \sim 30 \mathrm{~mm}$. Figure 9 shows the change of critical buckling load when $r_{2}$ changes. $r_{2}$ has a greater influence on $F_{c r, y}^{*}$, and the two are positively correlated. $r_{2}$ has little effect on $F_{c r, x}^{*}$, and the two are negatively correlated. When $r_{2}$ expands up and down by $50 \%, F_{c r, x}^{*}$ decreases by $1.93 \%$ and increases by $1.87 \%$. This is because $F_{c r, x}^{*}$ is significantly affected by the moment of inertia around the $o x$ axis. It can be seen from formula (5) that although $I_{x, 2}$ and $r_{2}$ are positively correlated, when $r_{2}$ increases, arc segment angle $\alpha$ will decrease (as shown in Figure 10), resulting in the overall moment of inertia around ox axis will decrease .

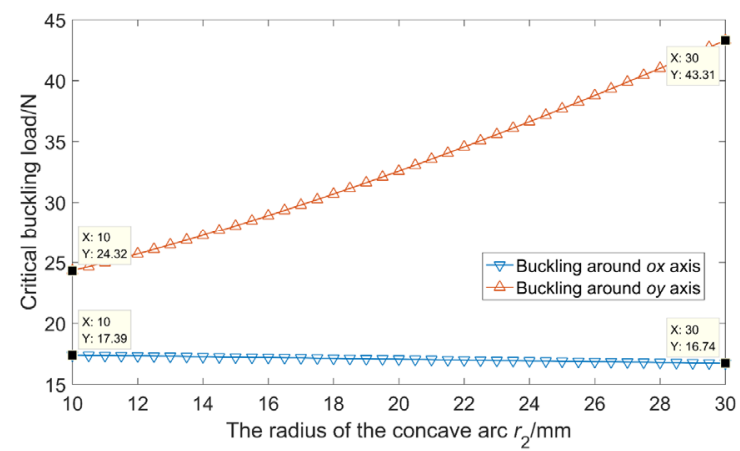

Fig. 9. The influence of $r_{2}$ on critical buckling load 


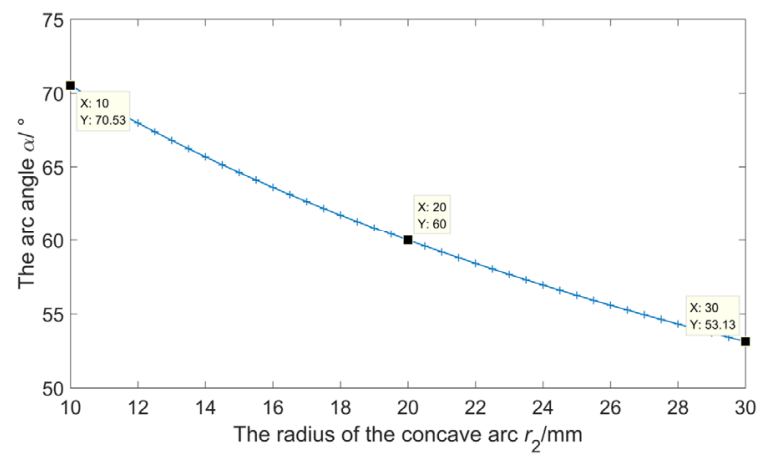

Fig. 10. The influence of $r_{2}$ on $\alpha$

\subsubsection{The influence of the center ordinate of convex arc on critical buckling load}

Based on the original parameters of the model, expanding the value of $y_{1}$ from $0 \mathrm{~mm}$ to $-10 \mathrm{~mm} \sim 10 \mathrm{~mm}$. Figure 11 shows the change of critical buckling load when $y_{1}$ changes. $y_{1}$ has a positive correlation with the critical buckling load. When $y_{1}$ increases to $10 \mathrm{~mm}$ and decreases to $-10 \mathrm{~mm}$, respectively, $F_{c r, x}^{*}$ increases to $47.98 \mathrm{~N}$ and decreases to $2.963 \mathrm{~N}$, and $F_{c r, y}^{*}$ increases to $48.25 \mathrm{~N}$ and decreases to $14.94 \mathrm{~N}$. As $y_{1}$ gradually increases, the slope of the relationship between $y_{1}$ and $F_{c r, x}^{*}$ gradually increases, and the slope of the relationship between $y_{1}$ and $F_{c r, y}^{*}$ gradually decreases. When $y_{1}$ increases to $10 \mathrm{~mm}$, the difference between $F_{c r, x}^{*}$ and $F_{c r, y}^{*}$ is only $0.56 \%$. If $y_{1}$ continues to increase, $F_{c r, x}^{*}$ will exceed $F_{c r, y}^{*}$. Therefore, $y_{1}$ has a more significant influence on $F_{c r, x}^{*}$.

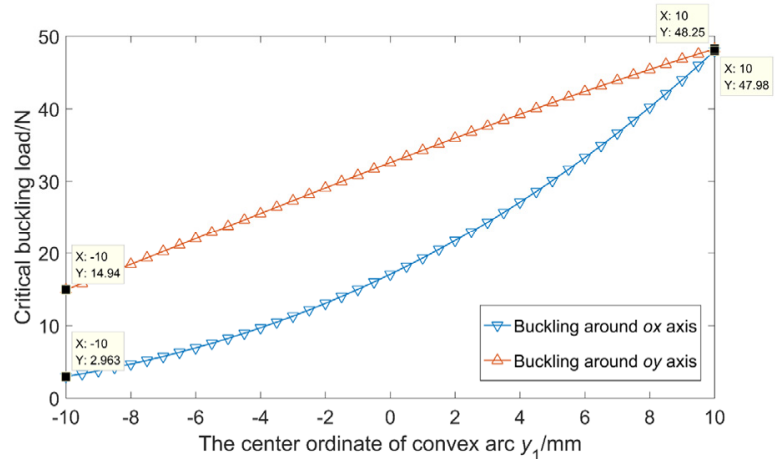

Fig. 11. The influence of $y_{1}$ on critical buckling load

\subsubsection{The influence of the length of straight section on critical buckling load}

Based on the original parameters of the model, expanding the value of $w$ from $5 \mathrm{~mm}$ to $2.5 \mathrm{~mm} \sim 7.5 \mathrm{~mm}$. Figure 12 shows the change of critical buckling load when $w$ changes. $w$ has a positive correlation with $F_{c r, y}^{*}$. When $w$ increases and decreases by $50 \%, F_{c r, y}^{*}$ increases by $16.24 \%$ and decreases by $14.36 \%$. $w$ has almost no effect on $F_{c r, x}^{*}$. This is because the distance between the straight section and the $o x$ axis is almost zero, so $w$ has almost no effect on the moment of inertia around the $o x$ axis. Therefore, the influence of $w$ on $F_{c r, x}^{*}$ is almost zero.

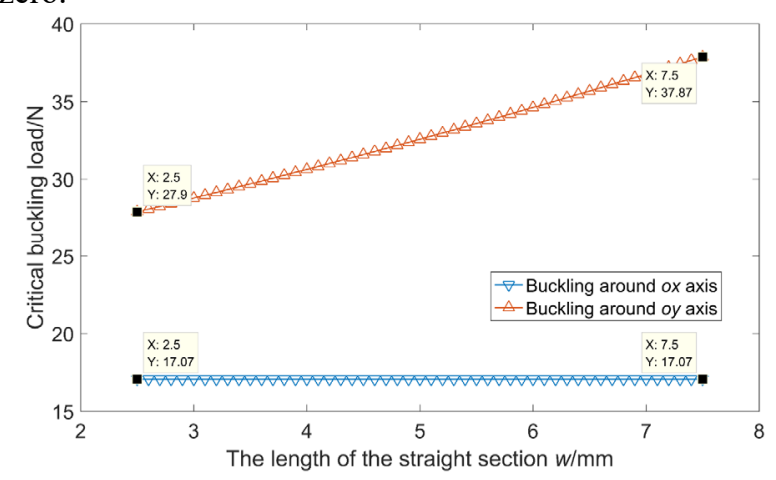

Fig. 12. The influence of $w$ on critical buckling load

\subsection{The influence of layer parameters on critical buckling load}

\subsubsection{The influence of the layer thickness on critical buckling load}

Based on the original parameters of the model, expanding the value of $t$ from $5 \mathrm{~mm}$ to $0.02 \mathrm{~mm} \sim 0.06 \mathrm{~mm}$. Figure 13 shows the change of critical buckling load when $t$ changes. $t$ and the critical buckling load are basically in a monotonically increasing linear relationship. When $t$ increases and decreases by $50 \%$, $F_{c r, x}^{*}$ increases by $49.97 \%$ and decreases by $50.02 \%$, and $F_{c r, y}^{*}$ increases by $49.94 \%$ and decreases by $50.03 \%$. Therefore, the critical buckling load can be increased by increasing $t$.

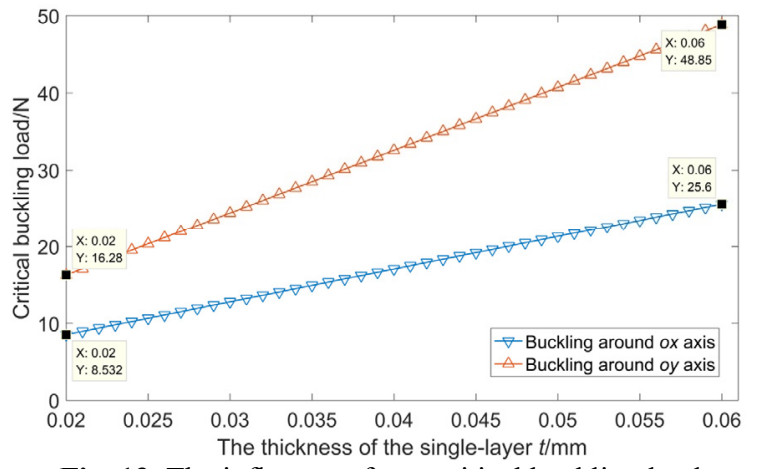

Fig. 13. The influence of $t$ on critical buckling load

\subsubsection{The influence of the layer angle on critical buckling load}

The layering method of the original model is limited to $\left[45^{\circ} /-45^{\circ} / \beta / 0 / \beta /-45^{\circ} / 45^{\circ}\right]$, where the third and fifth layers only exist in the convex arc, and the plyer angle of the two layers is represented by $\beta$. On the basis of the original parameters of the model, the value of $\beta$ is expanded from $0^{\circ}$ to $-90^{\circ} \sim 90^{\circ}$. The change of the critical buckling load when $\beta$ changes is shown in Figure 14. 
The relationship curve between $\beta$ and critical buckling load is symmetrical about $\beta=0^{\circ}$. When the $\beta=90^{\circ}$, $F_{c r, x}^{*}$ is reduced to $10.40 \mathrm{~N}$, and $F_{c r, y}^{*}$ is reduced to $29.80 \mathrm{~N}$. When $\beta=52^{\circ}$, the equivalent elastic modulus along the axis of the lenticular boom obtains the minimum value, and then $F_{c r, x}^{*}$ and $F_{c r, y}^{*}$ obtain the minimum values, which are $8.984 \mathrm{~N}$ and $29.22 \mathrm{~N}$, respectively. When $\beta=0^{\circ}$, the equivalent elastic modulus along the axis of the lenticular boom reaches the maximum value, and the critical buckling load reaches the maximum value. Therefore, the critical buckling load can be increased by setting $\beta$ to $0^{\circ}$.

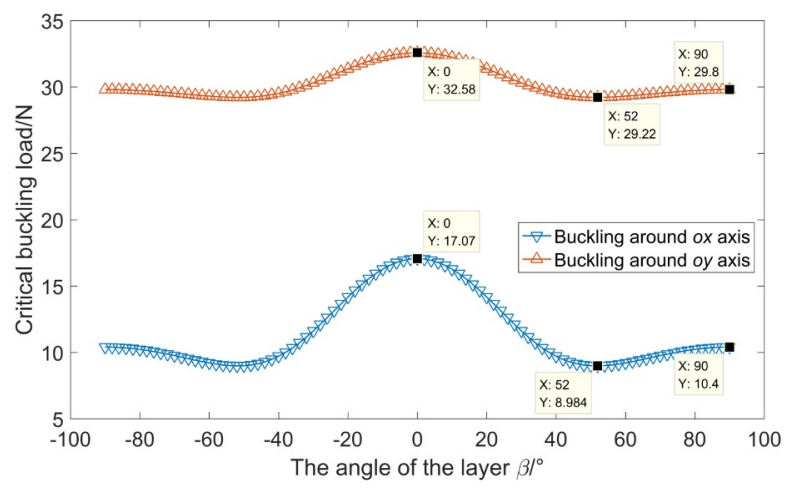

Fig. 14. The influence of $\beta$ on critical buckling load

\subsection{Comparative analysis of the influence law of each parameter}

The comparison chart of the influence law of each parameter is shown in Figure 15, and the variation range of each parameter is as described above. $r_{2}$ has little effect on $F_{c r, x}^{*}$ and the two are negatively correlated. $w$ has almost no effect on $F_{c r, x}^{*}$. The other parameters are positively related to the critical buckling load. Generally, $F_{c r, x}^{*}$ is smaller than $F_{c r, y}^{*}$, that is, the buckling around the $o x$ axis will take priority. Only when $y_{1}$ is large, the buckling around the oy axis will take priority. $r_{1}$ has the most significant influence on the critical buckling load. $r_{2}, y_{1}, w$ and $t$ have a greater impact on $F_{c r, y}^{*}$, while $\beta$ has a smaller impact on $F_{c r, y}^{*} \cdot y_{1}, t$ and $\beta$ have a greater influence on $F_{c r, x}^{*}$.

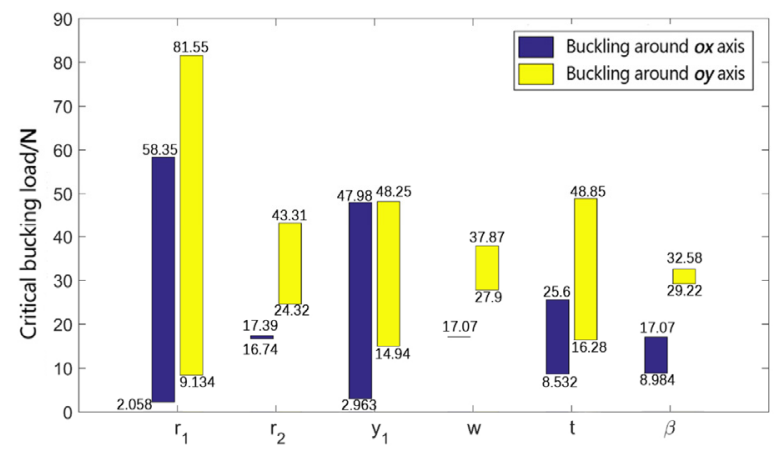

Fig. 15. Comparison chart of the influence law of each parameter

\section{Conclusion}

Aiming at the buckling problem of lenticular boom under axial compression, based on Euler's formula and laminated theory, this paper derives the critical buckling load formula of lenticular boom. The correctness of the formula is verified by finite element method, the applicable conditions are discussed. The influence of cross-section geometric parameters and layer parameters on its critical load is systematically analyzed. The main conclusions obtained are as follows:

(1) Based on Euler's formula and laminated theory, the critical buckling load formula of the lenticular boom is derived, and its correctness is verified by the finite element method. After discussion, it is concluded that the formula is only applicable to the lenticular boom with a slenderness ratio greater than 64. For the lenticular boom with small slenderness ratio, the error is larger;

(2) Generally, $F_{c r, x}^{*}$ is smaller than $F_{c r, y}^{*}$, that is, the buckling around the $o x$ axis will take priority. Only when $y_{1}$ is large, the buckling around the oy axis will take priority.

(3) Except for $r_{2}$ and $F_{c r, x}^{*}$ which are negatively correlated, the other parameters are positively correlated with $F_{c r, x}^{*}$ and $F_{c r, y}^{*}$, and $w$ has almost no influence on $F_{c r, x}^{*}$;

(4) $r_{1}$ has the most significant influence on the critical buckling load. $r_{2}, y_{1}, w$ and $t$ have a greater influence on $F_{c r, y}^{*}$, and $\beta$ has a smaller influence on $F_{c r, y}^{*} \cdot y_{1}, t$, and $\beta$ have a greater impact on $F_{c r, x}^{*}$, while $r_{2}$ has a small impact on $F_{c r, x}^{*}$.

\section{Acknowledgments}

This work is supported by the National Natural Science Foundation of China under the Grant No. 11702321.

\section{References}

1. Sickinger C, Herbeck L, Stroehlein T, et al. (2004) Lightweight deployable booms: design, manufacture, verification, and smart materials application. In: 55th International Astronautical Congress. Germany, DLR, 2054-2064.

2. Puig L, Barton A, Rando N. (2010) Review a review on large deployable structures for astrophysics missions. Acta Astronautica, 67(1-2): 12-26.

3. Wu J a B, Zhao Z A, Ren G A. (2013) Multibody analysis of the force in deploying booms. Journal of Guidance, Control, and Dynamics, 36(6): 1881-1885.

4. Sickinger C, Herbeck L, Breitbach E. (2006) Structural engineering on deployable CFRP booms 
for a solar propelled sailcraft. Acta Astronautica, 58(4): 185-196.

5. Herbeck L, Eiden M, Leipold M, et al. (2000) Development and test of deployable ultra-light weight CFRP boom for a solar sail. In: European Conference on Spacecraft Structures. Germany, DLR.

6. Hakkak F, Khoddam S. (2007) On calculation of preliminary design parameters for lenticular booms. Proceedings of the Institution of Mechanical Engineers. Part G: Journal of Aerospace Engineering, 221(3): 377-384.

7. Christian M. (2020) Buckling and post-buckling of thin-walled composite laminated beams - a review of engineering analysis methods. Applied Mechanics Reviews, 72(2): 020802,1-36.

8. Irwin R, Veen J V, Buchner-Santos E, et al. (2010) Low-mass deployable spacecraft booms. In: AIAA SPACE 2010 Conference \& Exposition. Anaheim, California.

9. $\mathrm{Yu} \mathrm{H}$, Wujun C, Ruixiong L, et al. (2016) Mechanical characteristics of deployable composite thin-walled lenticular tubes. Composite Structures, 153: 601-613.

10. Li R-X, Chen W-J, Fu G-Y. (2012) Buckling Analysis and Experiment of Lenticular CFRP ThinWalled Tube Space Boom under Axial Compression. Journal of Astronautics, 33(8): 1164-1170.(in Chinese)

11. Chu Z Y, Lei Y. (2014) Design theory and dynamic analysis of a deployable boom. Mechanism and Machine Theory, 71: 126-141.

12. Bai Jiangbo, Xiong J. (2014) Temperature effect on buckling properties of ultra-thin-walled lenticular collapsible composite tube subjected to axial compression. Chinese Journal of Aeronautics, 27(5): 1312-1317.

13. GUO Yizhu, YANG Haoyu, GUO Hongwei, LIU Rongqiang, LUO Ani. (2020) Analysis of mechanical properties of spatial thin-walled elastic extension bar. Journal of Harbin Institute of Technology, 52 (1): 107-112. (in Chinese)

14. Timoshenko S P, Gere J M. (1961) Theory of elastic stability. McGraw-Hill Book Company, New York.

15. Shen GL, Hu GK. (2006) Mechanics of Composite Materials. Tsinghua University Press, Beijing. (in Chinese)

16. Yu H, Wujun C, Jifeng G, et al. (2017) A study of flattening process of deployable composite thinwalled lenticular tubes under compression and tension. Composite Structures, 168: 164-177.

17. Luo S, Wang WB. (2020) Stability analysis of compressed spherical shell based on arc length method. Chinese Journal of Applied Mechanics, 37(1): 161-167, 479. 